\title{
Vigilancia de la resistencia de levaduras a antifúngicos
}

\author{
VÍCTOR SILVA V. ${ }^{1}$, M CRISTINA DÍAZ J. ${ }^{1}$, NALDY FEBRÉ ${ }^{2}$ y \\ RED DE DIAGNÓSTICO EN MICOLOGÍA MÉDICA ${ }^{3 *}$
}

\section{SURVEILLANCE OF ANTIFUNGAL DRUGS RESISTANCE IN YEASTS}

Invasive fungal infections have significantly increased over the last 20 years and, despite Candida albicans continues to be the main candida species, other species are emerging. Resistance to antifungal drugs is also emerging. We studied the resistance pattern to Amphotericin $B$ and azole agents in 110 blood or other normally sterile sites yeasts clinical isolates. Overall, 5\% resistance to azoles was detected and $100 \%$ were sensitive to Amphotericin B. The aim of this work is to review the trends in yeasts resistance from Chile and other countries, and to warrant the maintenance of a resistance fungal surveillance program.

Key words: Susceptibility, Antifungal drugs resistance, Invasive mycosis, Candida spp.

\section{INTRODUCCIÓN}

Las infecciones fúngicas invasoras han experimentado un aumento considerable en las últimas dos décadas, sobresaliendo las infecciones del torrente sanguíneo por especies del género Candida. ${ }^{1,2}$ La población susceptible consiste mayoritariamente en pacientes neutropénicos, prematuros e internados en UCI. Estas infecciones oportunistas son principalmente de origen nosocomial y la fuente es fundamentalmente endógena. ${ }^{3,4} \mathrm{El}$ género Candida está formado por 163 especies, de las cuales aproximadamente 10 son las responsables de las infecciones en el hombre, describiéndose a $C$. albicans como la especie más importante. ${ }^{5}$ Estos agentes son cosmopolitas y forman parte de la microbiota del hombre y animales, colonizando mucosas del tracto intestinal (50 a 70\%), boca (30 a 50\%), vaginal (5 a 30\%) y piel (4 a 7\%). Sin embargo, su presencia en manos del personal de salud es muy superior, con frecuencia de $20 \%$ en el personal médico y hasta $80 \%$ en el de enfermería. ${ }^{6,7}$ Recientemente, nuestro grupo demostró que la colonización de manos en estudiantes de medicina, aumenta significativamente según el período de estudio, siendo de $7 \%$ en el grupo básico, $19 \%$ en el preclínico y $30 \%$ en el clínico. Por otro lado, los alumnos de carreras distintas al área de la salud, presentaron 7 a $10 \%$ de colonización entre los distintos años de carrera. ${ }^{8}$

\footnotetext{
${ }^{1}$ Programa de Microbiología y Micología, ICBM, Facultad de Medicina, Universidad de Chile.

${ }^{2}$ Comité de Infecciones, Hospital del Tórax.

${ }^{3}$ Médicos y Tecnólogos Médicos de Laboratorios de Microbiología de la Red* en pág. S82.
} 


\section{Evolución de la candidiasis invasora}

A inicios de los años 60, en E.U.A. sólo se reportaban 48 casos de candidiasis invasora, siendo $C$. albicans la principal especie. ${ }^{9} \mathrm{Du}-$ rante la década de los ' 80 se describieron 25.000 infecciones nosocomiales del torrente sanguíneo en 124 hospitales americanos, relatándose aumento de candidemias de hasta $487 \%$ en hospitales universitarios de gran tamaño, con mortalidad atribuible de $29 \%$, siendo significativamente superior al $17 \%$ causado por patógenos bacterianos. ${ }^{10}$ Actualmente, en E.U.A. se considera a Candida spp como el primer y tercer agente de ITU y sepsis nosocomial, respectivamente. ${ }^{2}$ En nuestro país, a través del informe de infecciones intrahospitalarias del Ministerio de Salud (1999), se puede extraer que Candida spp es el tercer agente de ITU en UCI $(13,5 \%)$ y el cuarto agente de sepsis en pediatría $(7,5 \%)$, siendo el principal agente de sepsis asociada a nutrición parenteral en niños (30\%) y el cuarto en adultos con $10 \%$ de los totales estudiados. ${ }^{11}$

Además del aumento, se ha descrito un cambio en el patrón etiológico de estos cuadros (Tabla 1). ${ }^{12,13}$ A pesar de continuar C. albicans presentándose como la especie más frecuente en todos los tipos de candidiasis, su prevalencia ha disminuido drásticamente como agente de infecciones invasoras, dando lugar a un aumento considerable de otras especies del género. En los recientes informes se reporta una frecuencia estable de $C$. albicans, un aumento significativo de C. glabrata en diversas regio-

Tabla 1. Distribución de especies de Candida aisladas de sangre, según porcentaje de frecuencia y período de tiempo en E.U.A.

\begin{tabular}{|c|c|c|c|}
\hline Especie de Candida & $\begin{array}{c}1984 \\
(\%)\end{array}$ & $\begin{array}{c}1990 \\
(\%)\end{array}$ & $\begin{array}{c}1997 \\
(\%)\end{array}$ \\
\hline C. albicans & 80 & 58 & 56 \\
\hline C. tropicalis & 10 & 15 & 7 \\
\hline C. parapsilosis & 6 & 12 & 9 \\
\hline C. glabrata & & 9 & 19 \\
\hline C. krusei & $<9$ & & 2,5 \\
\hline C. guilliermondii & & $<6$ & 0,5 \\
\hline Otras especies & & & 6 \\
\hline
\end{tabular}

nes del globo, un aumento de $C$. parapsilosis, principalmente en población neutropénica y una disminución de C. tropicalis. ${ }^{14,15}$

\section{Susceptibilidad in vitro - método de referencia}

Debido a los innumerables problemas de concordancia en susceptibilidad antifúngica para levaduras, detectados en estudios multicéntricos, el National Committee for Clinical Laboratory Standards (NCCLS) publicó en 1997 el documento M27-A, que describe el método estándar de macro y microdilución, aprobados para determinar cuantitativamente la susceptibilidad in vitro para levaduras. El medio de cultivo es caldo RPMI 1640, tamponado a $\mathrm{pH} 7,0$ con MOPS; el inóculo es determinado mediante espectrofotometría a $540 \mathrm{~nm}$ para obtener una concentración final de $1.000 \mathrm{UFC} / \mathrm{ml}$, incubándose a $37^{\circ} \mathrm{C}$ durante 48 y 72 horas, para Candida spp y Cryptococcus neoformans, respectivamente. La determinación de la CIM en la macrodilución para anfotericina $\mathrm{B}$ se interpreta como la menor concentración del fármaco capaz de inhibir visualmente el crecimiento de la levadura, para los azoles y 5 fluocitocina (5-FC), el punto de corte es con inhibición del $80 \%$ del crecimiento. En la microdilución, la CIM para anfotericina $\mathrm{B}$ es determinada con la inhibición $>90 \%$ de crecimiento y en los azoles y 5-FC, con el 50\% de inhibición. ${ }^{16}$

Los métodos de macro y microdilución son iguales en principio; sin embargo, la microdilución presenta una serie de ventajas comparativas al requerir menor cantidad de materiales y medio, mayor facilidad y aplicabilidad con economía de espacio y menor costo. ${ }^{17} \mathrm{El}$ gran impacto de la estandarización del método de susceptibilidad in vitro para levaduras fue aumentar la concordancia entre laboratorios, además de universalizar la técnica de referencia. Rex et al presentaron en 1997 los criterios de susceptibilidad, resistencia y susceptibilidad dosis dependiente (SDD) de las levaduras frente a los antifúngicos, permitiendo detectar resistencia in vitro y ayudar a determinar falla clínica (Tabla 2). ${ }^{18}$ Sin embargo, la estanda- 
rización del método presenta limitaciones con la correlación clínica.

\section{Resistencia antifúngica en levaduras}

En los últimos años, se aisla cada vez con mayor frecuencia, cepas de levaduras con susceptibilidad disminuida o resistentes a los antifúngicos. ${ }^{19}$ La resistencia a antifúngicos puede ser clínica o in vitro, siendo la primera, consecuencia fundamental del bajo nivel del fármaco en el tejido y/o sangre, debido a una interacción entre fármacos o al severo grado de inmunodepresión del paciente. La resistencia in vitro puede ser primaria, donde la levadura es naturalmente resistente, siendo el mejor ejemplo la resistencia intrínseca que presenta C. krusei frente a fluconazol. También puede ser secundaria, donde cepas susceptibles se transforman en organismos resistentes debido al contacto previo con el antimicótico. Este último tipo de resistencia, inusual en el pasado, es hoy el más frecuente y se observa principalmente en cepas de C. glabrata. El consenso es que la resistencia antifúngica depende de la interacción entre el hospedero, el fármaco y el hongo; sin embargo, los factores del paciente son con frecuencia los más relevantes para definir resistencia. ${ }^{17,19}$

Con la introducción del fluconazol las candidemias por $C$. albicans y $C$. tropicalis disminuyeron. Sin embargo, las causadas por C. krusei y principalmente por C. glabrata experimentaron un aumento significativo. La mayoría de los relatos de resistencia en levaduras se refieren al fluconazol, en cepas aisladas de pacientes con SIDA. ${ }^{20}$ A partir de 1.579 cepas aisladas de candidemias entre 1992 y 1998 en E.U.A., se verificó que la resistencia en $C$. albicans fluctuaba entre 0 y $33 \%$ con una media de $1,4 \%$, siendo estos valores inferiores a los observados en cepas de $C$. noalbicans, cuyos rangos de resistencia fueron de 1,6 a $5,7 \%$ con una media de $3,3 \% .{ }^{21} \mathrm{La}$ primera cepa de Candida resistente a fluconazol aislada en paciente no infectado por VIH, presentó co-resistencia a otros azólicos. ${ }^{22}$ Este fenómeno se ha detectado principalmente en $C$. krusei y $C$. glabrata.

Recientemente, la red canadiense publicó sus datos de vigilancia entre 1996 y 1998, período en el cual aislaron 442 cepas de Candida de infecciones invasoras a partir de 51 hospitales. El $10 \%$ de los pacientes presentó tratamiento previo con azólicos y la frecuencia de $C$. noalbicans fue significativamente superior. Del total de cepas, detectaron $3 \%$ de resistencia a anfotericina $\mathrm{B}$, siendo estas cepas de $C$. noalbicans. Para itraconazol y fluconazol los porcentajes de resistencia variaron ampliamente según la especie, sobresaliendo $C$. glabrata con 13 y $9 \%$ de resistencia frente a cada antifúngico respectivamente. $C$. krusei mostró 7 y $13 \%$ de resistencia a estos fármacos,, respectivamente. ${ }^{15}$

Los mecanismos de resistencia propuestos son: disminución de la permeabilidad de la membrana citoplasmática, sobreexpresión de enzimas y mutaciones de compensación. Sin embargo, los mecanismos demostrados son cambios en la $\alpha$ lanosterol demetilasa, cambios en la D5-6 esterol desaturasa y bomba de eflujo. ${ }^{17,23}$

Tabla 2. Criterios de susceptibilidad de las levaduras frente a antifúngicos de administración sistémica

\begin{tabular}{lcccc}
\hline Criterios & $\begin{array}{c}\text { Anfotericina B } \\
(\boldsymbol{\mu} \mathbf{g} / \mathbf{m l})\end{array}$ & $\begin{array}{c}\text { Ketoconazol } \\
(\boldsymbol{\mu} \mathbf{g} / \mathbf{m l})\end{array}$ & $\begin{array}{c}\text { Itraconazol } \\
(\boldsymbol{\mu} \mathbf{g} / \mathbf{m l})\end{array}$ & $\begin{array}{c}\text { Fluconazol } \\
(\boldsymbol{\mu} \mathbf{g} / \mathbf{m l})\end{array}$ \\
\hline Susceptible & $<1$ & $<0,125$ & $<0,125$ & $<8$ \\
SDD* & $>2$ & 0,25 a 0,5 & $0,25-0,5$ & 16 a 32 \\
Resistente & $>1$ & $>1$ & $>64$ \\
$*$ Susceptibilidad dosis dependiente & & & \\
\hline
\end{tabular}




\section{Vigilancia de resistencia en Chile}

Nuestro grupo concretó en marzo del 2000 una Red de Diagnóstico en Micología Médica cuya primera actividad fue establecer un programa de vigilancia de las infecciones fúngicas invasoras en Chile, con la participación de 13 centros asistenciales; 9 de la Región Metropolitana (Santiago) y 4 de otras regiones. Los objetivos establecidos fueron:

- identificar las especies de hongos aislados de micosis invasoras, y

- determinar la susceptibilidad in vitro de las levaduras.

El aislamiento de cada cepa fue realizado en los laboratorios integrantes de la red, siendo derivada junto a la ficha epidemiológica a nuestro centro. La identificación de especie fue realizada utilizando el procedimiento estándar, que incluye estudio fisiológico, bioquímico y micromorfológico. ${ }^{5}$ La susceptibilidad antifúngica se determinó por el método estándar de microdilución, con las modificaciones aceptadas que incluyen incorporación de glucosa al $2 \%$ al medio RPMI 1640 y la lectura de CIM a las 24 y 48 horas con apoyo espectofotométrico a $540 \mathrm{~nm}$, lo cual facilita la determinación del punto de corte y la interpretación del examen. Como control de calidad se usaron las cepas ATCC control de C. krusei y C. parapsilosis, que presentan CIM conocidas para cada antifúngico y el control de crecimiento para cada cepa, considerándose aceptable una turbidez de crecimiento con densidad óptica $>$ a $350 .{ }^{16}$

\section{RESULTADOS}

Hasta marzo del 2001 se han incorporado cepas provenientes de 131 pacientes con micosis invasoras, de los cuales $121(92,4 \%)$ presentaron infecciones por levaduras y $10(7,6 \%)$ por hongos filamentosos. C. albicans sin superar el $50 \%$ y $C$. parapsilosis, son las dos especies más prevalentes, tanto en sangre como en el análisis del total de las muestras recibidas. La tercera especie más frecuente en candidemias fue $C$. tropicalis $(14,3 \%)$. Al observar el total de levaduras aisladas, vemos que $C$. tropicalis es tan frecuente como $C$. neoformans (11,6 y 10,7\%, respectivamente). Cabe resaltar la baja frecuencia de C. glabrata $(3,3 \%)$ y $C$. krusei $(1,1 \%)$ en sangre y la identificación poco común de Saccharomyces cerevisiae y Trichosporum mucoides a partir de hemocultivos (Tabla 3 ).

Tabla 3. Distribución de especies de levadura según número de cepas y sitio de aislamiento, incorporadas a la red

\begin{tabular}{|c|c|c|c|}
\hline Especie & $\begin{array}{c}\text { Sangre } \\
\text { n }(\%)\end{array}$ & $\begin{array}{c}\text { Otro sitio } \\
\text { n }(\%)\end{array}$ & $\begin{array}{l}\text { Total } \\
\text { n }(\%)\end{array}$ \\
\hline C. albicans & $43(47,3)$ & $11(36,7)$ & $54(44,6)$ \\
\hline C. famata & $6(6,6)$ & 0 & $6(5,0)$ \\
\hline C. guilliermondii & 0 & $1(3,3)$ & $1(0,8)$ \\
\hline C. glabrata & $3(3,3)$ & $2(6,7)$ & $5(4,1)$ \\
\hline C. kefyr & $1(1,1)$ & 0 & $1(0,8)$ \\
\hline C. krusei & $1(1,1)$ & 0 & $1(0,8)$ \\
\hline C. lusitaniae & 0 & $2(6,7)$ & $2(1,7)$ \\
\hline C. parapsilosis & $19(20,9)$ & $2(6,7)$ & $21(17,4)$ \\
\hline C. tropicalis & $13(14,3)$ & $1(3,3)$ & $14(11,6)$ \\
\hline C. neoformans & $2(2,2)$ & $11(36,7)$ & $13(10,7)$ \\
\hline S. cerevisiae & $2(2,2)$ & 0 & $2(1,7)$ \\
\hline T. mucoides & $1(1,1)$ & 0 & $1(0,8)$ \\
\hline Total & $91(100,0)$ & $30(100)$ & $121(100)$ \\
\hline
\end{tabular}

\section{S 152}


Vigilancia de la resistencia en levaduras - V. Silva V. et al

Tabla 4. Principales especies de Candida aisladas de sangre, según país

\begin{tabular}{lccrrrr}
\hline Especie & $\begin{array}{c}\text { E.U.A. } \\
(\boldsymbol{\%})\end{array}$ & $\begin{array}{c}\text { Canadá } \\
(\boldsymbol{\%})\end{array}$ & $\begin{array}{c}\text { Taiwan } \\
(\boldsymbol{\%})\end{array}$ & $\begin{array}{c}\text { Argentina } \\
(\boldsymbol{\%})\end{array}$ & $\begin{array}{c}\text { Brasil } \\
(\boldsymbol{\%})\end{array}$ & $\begin{array}{c}\text { Chile } \\
(\boldsymbol{\%})\end{array}$ \\
\hline C. albicans & 56 & 54 & 50 & 51 & 37 & 50 \\
C. glabrata & 19 & 15 & 14 & 2 & 4 & 4 \\
C. krusei & 3 & 3 & 3 & 3 & 0 & 1 \\
C. parapsilosis & 9 & 12 & 9 & 20 & 25 & 22 \\
C. tropicalis & 7 & 9 & 20 & 23 & 24 & 15 \\
Otras especies & 6 & 7 & 4 & 1 & 10 & 8 \\
\hline
\end{tabular}

Al comparar nuestros datos de candidemias con los de otros países, observamos que $C$. albicans es la especie mayormente prevalente, con una frecuencia cercana al 50\% (Tabla 4). Sin embargo, la segunda especie en frecuencia varía considerablemente, siendo C. parapsilosis en Chile, C. glabrata en E.U.A. ${ }^{13}$, C. tropicalis en Taiwan ${ }^{24}, C$. glabrata y $C$. parapsilosis en Canadá $^{15}, C$. parapsilosis y $C$. tropicalis en Argentina $^{25}$ y Brasil. ${ }^{26}$

$\mathrm{Al}$ determinar la susceptibilidad de las levaduras estudiadas en la red, verificamos que todas fueron sensibles a anfotericina B con CIM $<1 \mu \mathrm{g} / \mathrm{ml}$ (Tabla 5). Frente a los azólicos, se obtuvo $5 \%$ de resistencia y sobre $80 \%$ de susceptibilidad, siendo muy distinta la susceptibilidad según especie. C. albicans presentó 100 y $96 \%$ de susceptibilidad para itraconazol y fluconazol, respectivamente. Por otro lado, $C$. glabrata mostró 60 y $40 \%$ de resistencia frente a los mismos fármacos. Además, se detectó resistencia a fluconazol en cepas de $C$. tropicalis y $C$. parapsilosis. Es importante resaltar que las CIM $_{50}$ y CIM $_{90}$ de $C$. glabrata para itraconazol correspondían a valores considerados resistentes, al igual que la $\mathrm{CIM}_{90}$ para fluconazol de $C$. glabrata y $C$. tropicalis (Tablas 6 y 7). El fenómeno de co-resistencia fue determinado en una cepa de $C$. glabrata, quien mostró resistencia simultánea frente a fluconazol e itraconazol.

\section{Cuándo determinar la susceptibilidad in vitro de levaduras}

La identificación precoz de la especie de levadura aislada de la micosis invasora es de gran utilidad para el médico clínico, apoyándolo en la elección de la conducta terapéutica. Sin embargo, según algunos, hoy se hace necesario implementar métodos para evaluar la susceptibilidad antifúngica en la rutina de los laboratorios de microbiología, debido principalmente a

Tabla 5. Rango de CIM, $\mathrm{CIM}_{50}, \mathrm{CIM}_{90}$ y porcentaje de susceptibilidad de especies de levaduras frente a anfotericina $B$

\begin{tabular}{lrcccc}
\hline Especie & $\mathbf{N}$ & $\begin{array}{c}\text { Rango } \\
(\boldsymbol{\mu g} / \mathbf{m l})\end{array}$ & $\begin{array}{c}\mathbf{C I M}_{\mathbf{5 0}} \\
(\boldsymbol{\mu} \mathbf{g} \mathbf{m l})\end{array}$ & $\begin{array}{c}\mathbf{C I M}_{\mathbf{9 0}} \\
(\boldsymbol{\mu g} / \mathbf{m l})\end{array}$ & $\begin{array}{c}\text { Susceptibilidad } \\
(\boldsymbol{\%})\end{array}$ \\
\hline C. albicans & 52 & $0,013-1$ & 0,06 & 1 & 100 \\
C. famata & 6 & $0,03-0,13$ & 0,03 & 0,13 & 100 \\
C. glabrata & 5 & $0,06-1$ & 0,06 & 1 & 100 \\
C. parapsilosis & 21 & $0,03-1$ & 0,13 & 1 & 100 \\
C. tropicalis & 12 & $0,03-1$ & 0,03 & 0,25 & 100 \\
C. neoformans & 10 & $0,13-0,5$ & 0,25 & 0,5 & 100 \\
Total & 110 & $0,03-1$ & 0,06 & 0,5 & 100 \\
\hline
\end{tabular}


Vigilancia de la resistencia en levaduras - V. Silva V. et al

Tabla 6. Rango de CIM, $\mathrm{CIM}_{50} \mathrm{CIM}_{90}$ y porcentaje de susceptibilidad de especies de levaduras frente a itraconazol

\begin{tabular}{|c|c|c|c|c|c|c|c|}
\hline Especie & $\mathbf{n}$ & $\begin{array}{l}\text { Rango } \\
(\mu \mathrm{g} / \mathrm{ml})\end{array}$ & $\underbrace{\mathrm{CIM}_{50}}_{(\mu \mathrm{g} / \mathrm{ml})}$ & $\underset{(\mu \mathrm{g} / \mathrm{ml})}{\mathrm{CIM}_{90}}$ & $\begin{array}{c}\text { Susceptibilidad } \\
(\%)\end{array}$ & $\begin{array}{l}\text { SDD* } \\
(\%)\end{array}$ & $\begin{array}{c}\text { Resistencia } \\
(\%)\end{array}$ \\
\hline C. albicans & 52 & $0,03-0,06$ & 0,03 & 0,06 & 100 & 0 & 0 \\
\hline C. famata & 6 & $0,05-1$ & 0,5 & 1 & 0 & 67 & 33 \\
\hline C. glabrata & 5 & $0,03-16$ & 1 & 16 & 20 & 20 & 60 \\
\hline C. parapsilosis & 21 & $0,03-0,25$ & 0,03 & 0,13 & 95 & 5 & 0 \\
\hline C. tropicalis & 12 & $0,03-0,5$ & 0,03 & 0,5 & 83 & 17 & 0 \\
\hline C. neoformans & 10 & $0,03-0,5$ & 0,13 & 0,25 & 70 & 30 & 0 \\
\hline Total & 110 & $0,03-16$ & 0,03 & 0,5 & 83 & 12 & 5 \\
\hline
\end{tabular}

Tabla 7. Rango de CIM, $\operatorname{CIM}_{50} \operatorname{CIM}_{90}$ y porcentaje de susceptibilidad de especies de levaduras frente a fluconazol

\begin{tabular}{|c|c|c|c|c|c|c|c|}
\hline Especie & $\mathbf{n}$ & $\begin{array}{c}\text { Rango } \\
(\mu \mathrm{g} / \mathrm{ml})\end{array}$ & $\underset{(\mu \mathrm{g} / \mathrm{ml})}{\mathrm{CIM}_{50}}$ & $\begin{array}{l}\mathrm{CIM}_{90} \\
(\mu \mathrm{g} / \mathrm{ml})\end{array}$ & $\begin{array}{c}\text { Susceptibilidad } \\
(\%)\end{array}$ & $\begin{array}{c}\text { SDD } * \\
(\%)\end{array}$ & $\begin{array}{c}\text { Resistencia } \\
(\%)\end{array}$ \\
\hline C. albicans & 52 & $0,25-16$ & 0,25 & 2 & 96 & 4 & 0 \\
\hline C. famata & 6 & $8-16$ & 8 & 16 & 50 & 50 & 0 \\
\hline C. glabrata & 5 & $0,5-128$ & 16 & 128 & 40 & 20 & 40 \\
\hline C. parapsilosis & 21 & $0,25-128$ & 1 & 4 & 95 & 0 & 5 \\
\hline C. tropicalis & 12 & $0,25-128$ & 0,5 & 64 & 83 & 0 & 17 \\
\hline C. neoformans & 10 & $0,5-16$ & 2 & 8 & 90 & 10 & 0 \\
\hline Total & 110 & $0,25-128$ & 0,5 & 16 & 88 & 7 & 5 \\
\hline
\end{tabular}

los antecedentes de resistencia y a la introducción al arsenal terapéutico de nuevos antifúngicos.

Existe consenso para determinar la susceptibilidad in vitro en toda levadura aislada de:

- Candidiasis oro-faríngea en paciente infectado con VIH

- Micosis invasora

- Vaginitis recurrente

- Brotes nosocomiales

- Paciente bajo profilaxia antifúngica, fundamentalmente en neutropénicos.

El método ideal para determinar la susceptibilidad in vitro aún no ha sido desarrollado, ya que debe tener las siguientes características: ser reproducible, fácil, económico, rápido, buena correlación clínica, definición clara de los puntos de cortes y que requiera pocos equipos.
El método estándar, debido a diversas dificultades, no es aplicable en nuestra rutina microbiológica. Por tal motivo es recomendable que los laboratorios se apoyen en los métodos comerciales disponibles en el mercado, previo análisis de sus ventajas y de los estudios de correlación con el método de referencia. Los métodos comerciales más conocidos son Etest ${ }^{\circledR}$ (AB Biodisk-Suecia), Neosensitab ${ }^{\circledR}$ (Rosco-Dinamarca), Fungifast ${ }^{\circledR}$ (Sanofi PasteurFrancia), ATB Fungus ${ }^{\circledR}$ (BioMérieux-Francia), Asty ${ }^{\circledR}$ (Kyocuto Pharmaceutical Industries-Japón) y Sensitive Yeast One Panel ${ }^{\circledR}$ (Trek Diagnostic System-E.U.A.).

En conclusión, podemos citar la existencia cosmopolita de resistencia antifúngica en levaduras. C. albicans y C. parapsilosis son las dos principales especies de levaduras aisladas 
de micosis invasoras en Chile. En las cepas analizadas, se detectó $100 \%$ de susceptibilidad a anfotericina B y $5 \%$ de resistencia, tanto para itraconazol como fluconazol. C. glabrata es la especie preocupante en relación a susceptibilidad antifúngica, a pesar que existen algunas cepas de $C$. tropicalis y $C$. parapsilosis resistentes a fluconazol. Finalmente, los datos mostrados en esta publicación nos ratifican la necesidad de mantener una vigilancia nacional que nos permita monitorear la distribución y susceptibilidad antifúngica entre las levaduras aisladas de infecciones invasoras a través de la Red de Diagnóstico Micológico; ello contribuirá al manejo de estas micosis y a evaluar la evolución de resistencia en nuestro medio.

\section{RESUMEN}

Las infecciones fúngicas invasoras han aumentado en forma explosiva en los últimos 20 años. Desde la década de los '90 se ha observado un cambio continuo en el patrón etiológico de estas micosis, donde las especies del género Candida son los principales agentes. Además, en los últimos años se evidencia cada vez con mayor frecuencia, el aislamiento de levaduras menos sensibles o resistentes a los antifúngicos. El objetivo de la presente publicación es revisar los antecedentes de resistencia en levaduras, tanto en el exterior como en nuestro medio y enfatizar la necesidad de mantener una red de vigilancia de resistencia.

Médicos y Tecnólogos Médicos de

Laboratorios de Microbiología de la red:

- Asociación Chilena de Seguridad: Eugenio Reyes.

- Hospital Barros Luco Trudeau: Marcela San Martín, Carolina Allende, Angélica Aranda, Dina Concha, Sara Cisternas, Herta Barría, Liliana Paredes.

- Hospital Base de Valdivia: M. Emilia Arce.

- Hospital Carlos Van Buren: Ema Gaete, Gerardo Peralta.

- Centro de Diagnóstico Universidad Católica de Chile: Ana M. Guzmán, E. Pilar León.

- Centro de Diagnóstico y Tratamiento Eloísa Díaz: Maggie Vecchiola, Carmen Villalobos.

- Hospital Clínico Universidad de Chile: Mailing Chang, Marcela Castillo.

- Hospital Gustavo Fricke de Viña del Mar: Alejandro Joyas, Berta Ossandón, Patricia Tapia.

- Hospital Juan Noé de Arica: Ricardo Zepeda, Pablo Herrera.
- Hospital Luis Calvo Mackenna: M. Teresa Siri, Mónica Córdova.

- Hospital Militar del General Luis Felipe Brieva Arán: Gloria Zenteno, Beatriz Bórquez.

- Hospital San Juan de Dios: Mónica Lafourcade, Angel Geraldo.

- Hospital Sótero Del Río: Patricia González, Marcela Gaete.

Agradecimientos: A los asesores de la red: Naldy Febré, Eduardo Piontelli, Luis Thompson M y Luis Zaror, a los colegas, alumnos y técnicos que han colaborado desinteresadamente en este Programa de Vigilancia.

\section{BIBLIOGRAFÍA}

1.- JARVIS W R, MARTONE W J. Predominante pathogens in hospital infections. J Antimicrob Chemother 1992; 29: 19-24.

2.- RICHARDS M J, EDWARDS J R, CULVER D H, GAYNES R P and National Nosocomial Infections Surveillance System. Nosocomial infections in medical intensive care units in the United States. Crit Care Med 1999; 27: 887-92.

3.- WEY S B, MOTOMI M, PFALLER M A, WOOLSEN R F, WENZEL R P. Hospital acquired candidemia: the attributable mortality and excess length of stay. Arch Intern Med 1988; 148: 2642-7.

4.- BARRIOS-PEREZ A, MARTIN-RUIZ J L, JANNONE-FORES R et al. Prospective study of nosocomial infections in a medical UCI. A proposal for the generalized use of the National Nosocomial Infections Surveillance System rates. Rev Clin Esp 1998; 198: 284-8.

5.- KURTZMAN C P, FELL J W. The yeasts. A taxonomic study. 4ed. Elsevier, Amsterdam 1998; pp 1-1055.

6. BODEY G P. Candidiasis. Pathogenesis, Diagnosis and Treatment. 2nd. Raven Press, New York, 1993.pp $1-420$.

7.- STRAUSBAUGH L S, SEWELL D L, WARD T T, PFALLER MA, HEITZMAN T, T JOELKER R. High frequency of yeast carriage on hands of hospitalar personnel. J Clin Microbiol 1994; 32: 2299-300.

8.- SILVA V, ZEPEDA G, RYBAK M E. Prevalencia de levaduras en manos de estudiantes de medicina. Libro de Resúmenes XVII Congreso Chileno de Infectología. 9-12 de agosto, 2000, Viña del Mar: P-33.

9.- HURLEY R. Acute disseminated (septicemic) moniliasis in adults and children. Postgrad Med 1964; 40: 644-5.

10. BANERJEE S N, EMORI T G, CULVER D H et al. Secular trends in nosocomial primary bloodstream infections in the United States, 1980-1989. Am J Med 1991; 91: 86-9.

11.- OTAÍZA F, BRENNER P. Informe de la vigilancia epidemiológica de las infecciones intrahospitalarias, Chile-1998. Ministerio de Salud de Chile, 1999. 
12.- PFALLER M A, WENZEL R. Impact of the changing epidemiology of fungal infections in the 1990s. Eur J Clin Microbiol 1992; 11: 287-91.

13.- PFALLER M A, JONES R N, DOERN G V et al for SENTRY. Participant Group. International surveillance of bloodstream infections due to Candida species: Frequency of occurrence and antifungal susceptibilities of isolates collected in 1997 in the United States, Canada and South America for the SENTRY program. J Clin Microbiol 1998; 36: 1886-9.

14.- PFALLER M A, JONES R N, DOERN G V et al. Bloodstream infections due to Candida species: SENTRY antimicrobial surveillance program in North America and Latin America, 1997-1998. Antimicrob Agents Chemother 2000; 44: 747-51.

15.- ST-GERMAIN G, LAVERDIERE M, PELLETIER $\mathrm{R}$ et al. Prevalence and antifungal susceptibility of 442 Candida isolates from blood and other normally sterile sites: Results of a 2 year (1996 to 1998) multicenter surveillance study in Quebec, Canada. J Clin Microbiol 2001; 39: 949-53.

16.- NATIONAL COMMITTEE FOR CLINICAL LABORATORY STANDARDS. Reference method for broth dilution antifungal susceptibility testing of yeasts. Approved standard. Document M27-A. Wayne, PA: National Committee for Clinical Laboratory Standards, 1997.

17.- ESPINEL-INGROFF A, WARNOCK D W, VAZQUEZ J A et al. In vitro antifungal susceptibility methods and clinical implications of antifungal resistance. Med Mycol 2000; 38: 293-304.

18.- REX J H, PFALLER M A, GALGIANI J N et al. Development of interpretative breakpoint for antifungal susceptibility tests: Conceptual framework and analysis of in vitro-in vivo correlation date for fluconazole, itraconazole, and Candida infections. Subcommittee on antifungal susceptibility testing of the National Committe for Clinical Laboratory Standards. Clin Infect Dis 1997; 24: 235-47.

19.- FEBRÉ N, SILVA V, MEDEIROS E A S et al. Microbiological characteristics of yeast isolated from urinary tracts of intensive care unit patients undergoing urinary catheterization. J Clin Microbiol 1999; 37: 1584-6.

20.- SOBEL J D, OHMIT S E, SCHUMAN P et al. The evaluation of Candida species and fluconazole susceptibility among oral and vaginal isolates recovered from human immunodeficiency virus (HIV)seropositive and at-risk HIV-seronegative women. J Infect Dis 2001; 183: 286-93.

21.- PFALLER M A, MESSER S A, HOLLIS R J et al. Trends in species distribution and susceptibility to fluconazole among bloodstream isolates of Candida species in the United States. Diagn Microbiol Infect Dis 1999; 33: 217-22.

22. SOBEL J D, VAZQUEZ J A. Symptomatic vulvovaginitis due to fluconazole resistant Candida albicans in a female who was not infected with human immunodeficiency virus. Clin Infect Dis 1996; 22: 726-7.

23.- PARKINSON T, FALCONER D J, HITCHCOCK C A. Fluconazole resistance due to energy dependent drug efflux in Candida glabrata. Antimicrob Agents Chemother 1995; 39 (8): 1696-9.

24.- HUNG C C, CHEN Y C, CHANG S C et al. Nosocomial candidemia in a university hospital in Taiwan. J Formos Med Assoc 1996; 95: 19-28.

25.- RODERO L, DAVEL G, CÓRDOBA S, SORIA M, CANTEROS C, HOCHENFELLMER C. Multicenter study on nosocomial candidasis in the Republic of Argentina. Rev Argent Microbiol 1999; 31: 114-9.

26.- COLOMBO A L. Epidemiology and treatment of hematogenous candidiasis: A brazilian perspective. Braz J Infect Dis 2000; 4: 113-8.

Correspondencia a:

Víctor Silva V.

E-mail:vsilva@machi.med.uchile.cl 